\title{
Single Molecule Force Measurements of Perlecan/HSPG2: A Key Component of the Osteocyte Pericellular Matrix
}

Sithara S. Wijeratne ${ }^{1}$, Jerahme R. Martinez ${ }^{2}$, Brian J. Grindel ${ }^{2}$, Eric W. Frey ${ }^{1}$, Jingqiang Li ${ }^{1}$, Liyun Wang ${ }^{4}$, Mary C. Farach-Carson ${ }^{2,3,+}$, Ching-Hwa Kiang ${ }^{1,3}$

${ }^{1}$ Department of Physics and Astronomy, Rice University, Houston, TX 77005, USA

${ }^{2}$ Department of BioSciences, Rice University, Houston, TX 77005, USA

${ }^{3}$ Department of Bioengineering, Rice University, Houston, TX 77005, USA

${ }^{4}$ Department of Mechanical Engineering, University of Delaware, Newark, DE, USA

Sithara S. Wijeratne and Jerahme R. Martinez contributed equally to this work.

+To whom correspondence should be addressed:

Mary C. Farach-Carson, Ph.D.

Department of BioSciences

Rice University

BioScience Research Collaborative

6100 Main Street MS 650

Tel: (713) 348-5052

Email: farachca@rice.edu 


\section{Abstract}

Perlecan/HSPG2, a large, monomeric heparan sulfate proteoglycan (HSPG), is a key component of the lacunar canalicular system (LCS) of cortical bone, where it is part of the mechanosensing pericellular matrix (PCM) surrounding the osteocytic processes and serves as a tethering element that connects the osteocyte cell body to the bone matrix. Within the pericellular space surrounding the osteocyte cell body, perlecan can experience physiological fluid flow drag force and in that capacity function as a sensor to relay external stimuli to the osteocyte cell membrane. We previously showed a reduction in perlecan secretion alters the PCM fiber composition and interferes with bone's response to mechanical loading in vivo. To test our hypothesis that perlecan core protein can sustain tensile forces without unfolding under physiological loading conditions, atomic force microscopy (AFM) was used to capture images of perlecan monomers at nanoscale resolution and to perform single molecule force measurement (SMFMs). We found that the core protein of purified full-length human perlecan is of suitable size to span the pericellular space of the LCS, with a measured end-to-end length of $170 \pm 20$ $\mathrm{nm}$ and a diameter of 2-4 $\mathrm{nm}$. Force pulling revealed a strong protein core that can withstand over $100 \mathrm{pN}$ of tension well over the drag forces that are estimated to be exerted on the individual osteocyte tethers. Data fitting with an extensible worm-like chain model showed that the perlecan protein core has a mean elastic constant of $890 \mathrm{pN}$ and a corresponding Young's modulus of $71 \mathrm{MPa}$. We conclude perlecan has physical properties that would allow it to act as a strong but elastic tether in the LCS.

Keywords: perlecan, heparan sulfate proteoglycan, atomic force microscopy, osteocytes, lacunar canalicular system, bone 


\section{Introduction}

The basement membrane (BM) protein perlecan [1], also known as heparan sulfate proteoglycan 2 (HSPG2), is one of the largest $(470-700 \mathrm{kDa})$ and oldest ( $>550$ million years) matrix molecules with many important functions [2,3] . One of the ancient key functions of perlecan is to maintain the integrity of functional borders separating tissues and tissue layers through its long ( 200 nm) [4] and five modular core protein domains that interact with various matrix molecules [2]. Perlecan's modular structure (as shown in Fig. 1) has been retained throughout millions of years of evolution [2,5], and the largest portion of perlecan, domain IV, is about $90 \%$ identical across mammalian species [2]. The perlecan gene, HSPG2, can be found in early simple metazoan organisms with few cell layers and in developing and adult tissue borders in mammals as well. These tissue borders represent functional barriers such as those between extracellular matrix (ECM) and blood (e.g., glomerular basement membrane (BM)) [6], among muscle fibers [7], and at the osteochondral junctions of long bones [8]. Perlecan contributes to the structural stability of BM by binding to and linking together the individual laminin and collagen networks [9], which is critical for kidney's filtration function. Although initially identified as a structural component in BM, regulatory functions of perlecan have been recognized through the interactions of its attached heparan sulfate glycosaminoglycan (GAG) side chains with growth factors and other matrix proteins [4]. This newly gained evolutionary function for perlecan is especially prominent in tissues that typically experience mechanical loading, such as muscle, cartilage and bone tissue. In skeletal muscle tissue, perlecan maintains fast muscle mass and fiber composition in response to overloading [10]. In perlecan deficient slow-twitch soleus muscles, unloading resulted in loss of muscle mass through autophagy by the mTORC1 pathway [11]. Recent studies demonstrated that the perlecan-rich pericellular matrix (PCM) surrounding chondrocytes in articular cartilage exhibits distinct mechanical properties from the territorial ECM and modulates chondrocytes responses to mechanical stimuli via binding and release of FGF-2 [12-14]. More recently, our group identified 
the presence of perlecan in another functional interface, i.e., osteocyte lacunar-canalicular system (LCS) and its involvement in osteocyte-mediated mechanosensing in response to loadinduced fluid flow $[15,16]$.

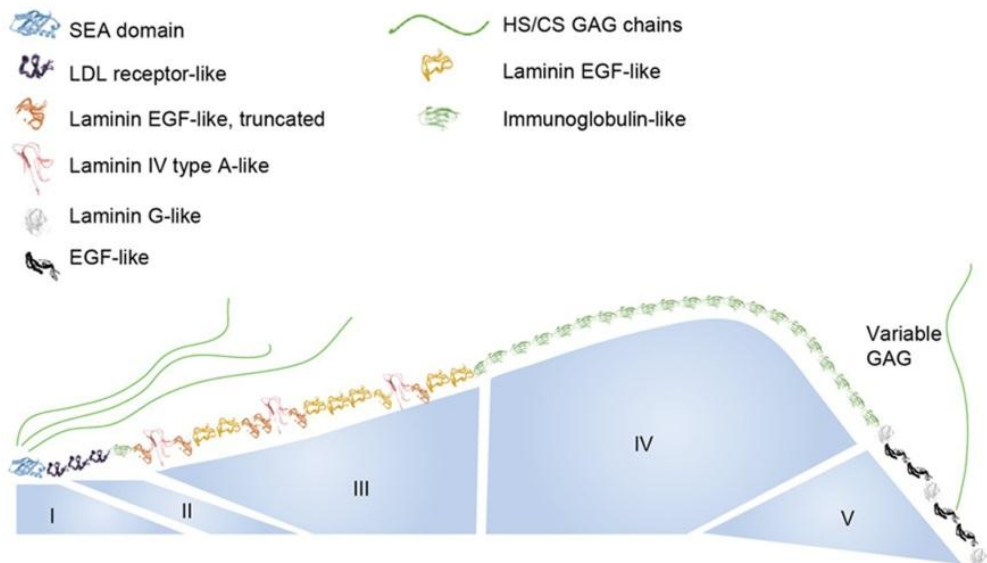

Figure 1. Schematic representation of the domain organization of human perlecan. Five structural domains are depicted as a strand of individually folded modules that share homology to various protein families. The entire molecule is 4391 amino acids with a core molecular mass approximately $470 \mathrm{kDa}$. The core protein, excluding the GAG side chains, is predicted to span $100-200 \mathrm{~nm}$ in length. With the addition of GAG chains (each 10-100 kDa or 20-150 nm) in domains I and $\mathrm{V}$ the molecule's dimensions can be extended.

Interstitial fluid flow in the bone LCS functions as a major mechanical stimulus that drives various cellular processes during bone adaptation [17]. Being the most abundant bone cells and strategically positioned in the middle of mineralized matrix, osteocytes serve as the primary sensors to perceive external mechanical signals through interstitial fluid flow $[18,19]$. For the past two decades, advances from in vitro and in vivo studies have elucidated multiple mechanotransduction pathways (e.g., Wnt/SOST and OPG/RANKL) for osteocytes to regulate the functions of osteoblasts and osteoclasts and thus orchestrate bone's response to mechanical stimuli (see recent reviews [20-22]). However, the mechanosensing apparatus that allows osteocyte to detect interstitial fluid remained unclear. Although a fibrous PCM containing proteoglycans and transverse tethers were hypothesized to surround the osteocytes in the bone LCS by Weinbaum and coworkers in $[18,23]$, respectively, the chemical composition of the PCM 
and the tethering candidates were not identified until ten years later. In 2011, we confirmed perlecan to be an important component of the osteocyte PCM, where reduced expression of perlecan results in fewer tethering elements within the pericellular space and narrower canalicular channels [15]. Furthermore, we demonstrated a 30\% reduction of the PCM fiber density and the lack of anabolic responses to in vivo mechanical loading using a perlecan deficient mouse [16]. Based on these in vivo results and the known properties of perlecan, we hypothesized that the perlecan-containing PCM tethers serve as flow sensors in the bone LCS and the fluid drag forces experienced by the PCM tethers were predicted to be at piconewton levels under physiological loading conditions [16]. The question remains as to whether perlecan molecule can withstand the predicted fluid drag forces in the bone LCS.

Although recognized as a key structural component of many territorial and pericellular matrices, perlecan's mechanical properties have yet to be explored. Given perlecan's linear modular structure, we considered it an ideal candidate for single molecule force measurements (SMFMs) using atomic force microscopy (AFM). This method has been widely adopted to study the mechanical strengths of individual proteins, ligand-receptor interactions, and large protein complexes [24]. Selective examples include investigations of mechanical properties of proteins involved in cytoskeleton rearrangement [25-28], tissue elasticity and ECM integrity [29,30]. In particular, SMFM by AFM has been very useful in elucidating the mechanical behaviors of large modular proteins containing tandem repeating motifs including a long stretch of contiguous immunoglobulin $(\mathrm{lg})$ modules such as titin [25,26,31]. The ability of titin to resist stretching and/or bending under mechanical force was demonstrated using SMFMs [32,33]. Perlecan contains Ig repeats in domain IV Fig. 1, similar to titin, but the abilities of perlecan to provide mechanical stability and flexibility to perlecan-rich tissues and their borders have not been measured. 
Our work here sought to investigate perlecan's mechanical properties to understand how perlecan might serve as an osteocyte PCM mechanosensing tether that endures physiological fluid flow drag within the bone LCS. We hypothesized that the perlecan core protein sustains tensile forces without unfolding under physiological loading conditions. For these studies, we carried out AFM imaging and force measurements on purified full-length perlecan with and without its GAG sidechains. While perlecan was engaged with the AFM tip and the tip was moved away under a controlled fashion, we recorded the tensile force experienced by perlecan as a function of extension length. The force-extension curves were fit with a worm-like chain model to determine perlecan's mechanical properties. Our objective was to demonstrate that the physical properties of perlecan are consistent with its proposed mechanosensing role in the bone LCS.

\section{Results}

\subsection{Perlecan morphology by AFM imaging}

Purified perlecan was isolated from the conditioned media of HT29 cells, a human colon carcinoma cell line, following previously established protocols [34] (Fig. 2 A). AFM images showed single isolated perlecan monomer structures (Fig. 2 B). Although protein aggregates were observed on occasion, single monomers were clearly visible. The core protein contained globular features having diameters of $2-4 \mathrm{~nm}$ and heights of $5-10 \mathrm{~nm}$. 


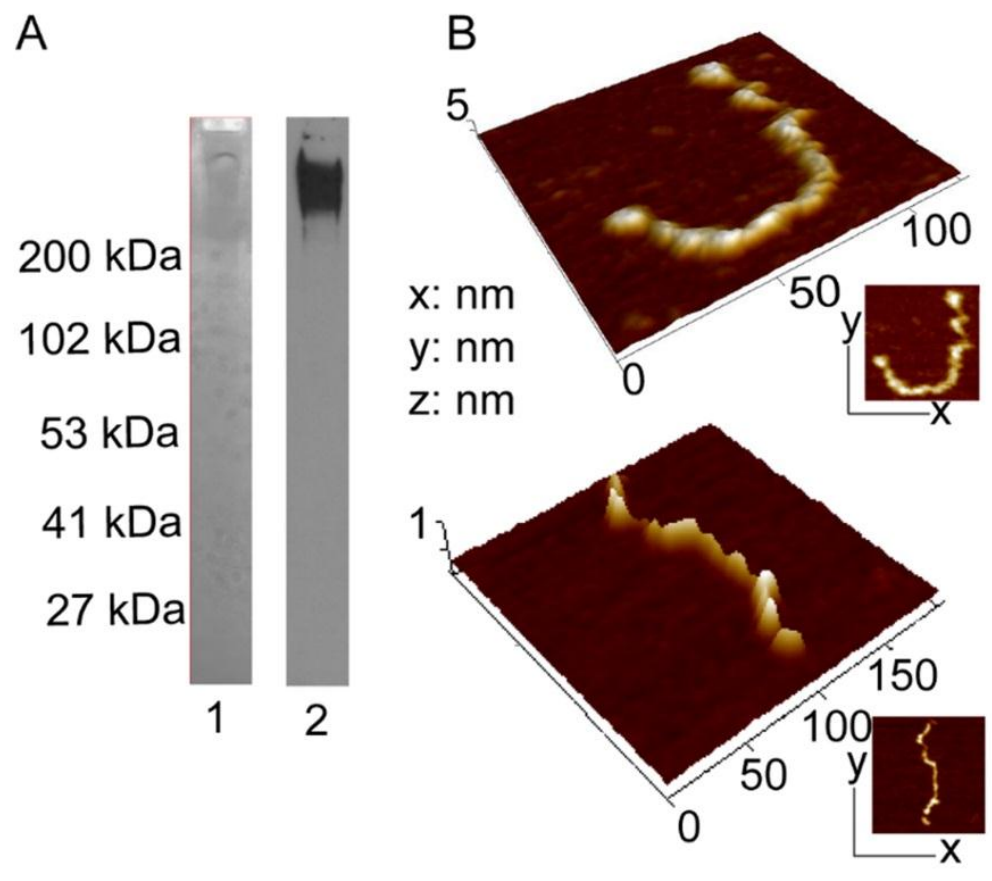

Figure 2. Three-dimensional AFM images of single perlecan molecules. (A) Lane 1 is a Coomassie stain and lane 2 is a perlecan western blot (Dm IV antibody 3135) of purified perlecan. (B) Single molecules exhibited a beads-on-a-string appearance with an extended linear- shaped conformation. Globular features were observed, possibly corresponding to protein modules. Perlecan molecules were measured to have diameters of $2-4 \mathrm{~nm}$ and heights of $5-10 \mathrm{~nm}$.

Single linear molecules were measured from end-to-end to determine a contour length (Fig. 3 A). The perlecan contour was converted to digitized coordinates to obtain accurate measurements (Fig. 3 B). A distribution of perlecan contours revealed a most common extension of $\sim 170 \pm 20 \mathrm{~nm}$ in length (Fig. 3 C). Persistence length was determined by plotting the cosine correlation function against the contour separation (Fig. $3 D$ ). A high correlation $(\sim 1)$ exists when two different coordinates have tangent vectors with similar angles, as one would expect in a straight or rigid molecule. On the other hand, when the tangents are pointing in opposite directions, as occurs in a curved region (Fig. $3 B$ ), the correlation is lower $(<1)$. The plot in Fig. $3 D$ shows that the cosine correlation for perlecan decreases the further away two points are from one another. Overall, perlecan molecules favored a long thin flexible 
conformation with a $L_{p}=19 \mathrm{~nm} \pm 5 \mathrm{~nm}$ (Eq. 1). Image analysis was attributed to the perlecan core itself, because GAGs were not consistently visible under the conditions used to optimally image core protein structures.
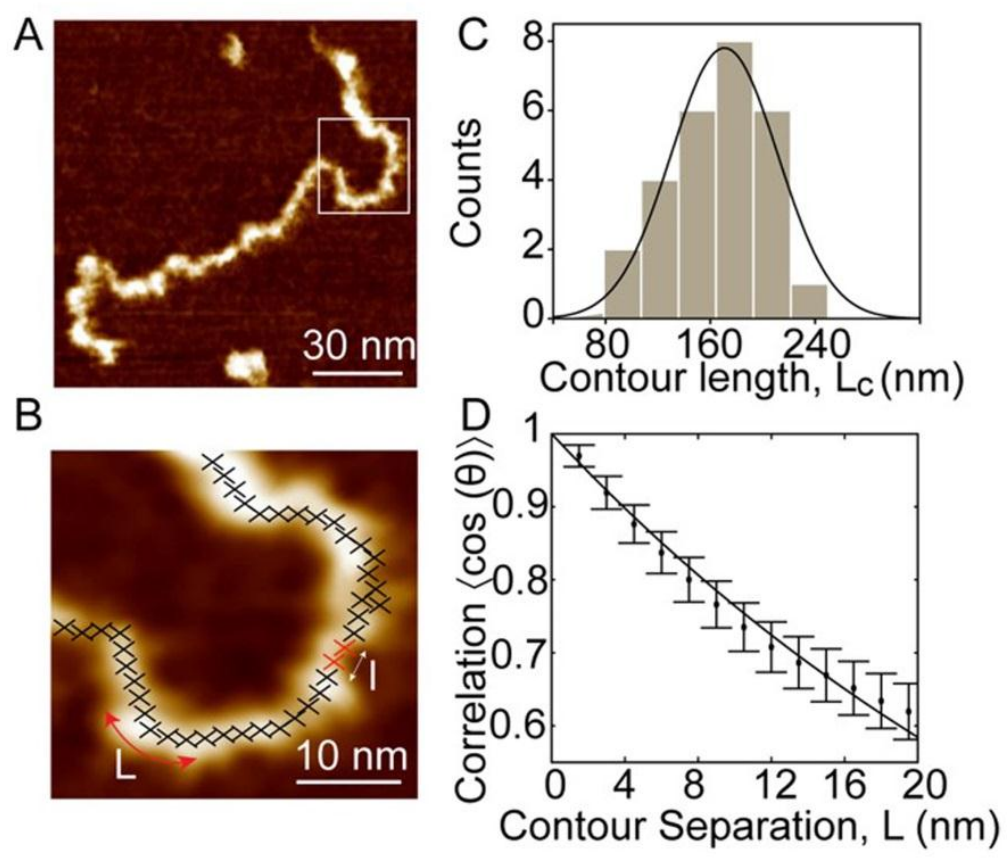

Figure 3. Analysis of the contour and persistent lengths of perlecan. (A) A representative AFM image of full-length perlecan used in the analysis (total 27 images). (B) An enlarged zoomin image of the box in $(A)$, showing the digitized contour tracing of the molecule used to find the contour length, $L_{c}(\mathrm{C})$. Histogram showing the distribution of the $L_{c}$ is fitted to a Gaussian curve (solid line); and the peak of the Gaussian is $170 \mathrm{~nm}$ with a standard deviation of $20 \mathrm{~nm}$. (D) Cross-correlation function $\langle\cos (\theta)\rangle$ as a function of contour separation. The data were fitted to Eq. 1 to obtain persistence length $L_{p}=19 \mathrm{~nm}$ (as the $\left.\langle\cos (\theta)\rangle=e^{-1 / 2}=0.6\right)$.

\subsection{Perlecan Elastic Properties by AFM Pulling}

The AFM pulling scheme we used is depicted in Fig. 4 A. While perlecan molecules remained attached to the gold substrate, randomly chosen segments of the molecule, which could be either core protein or GAG, were located then pulled upon using the AFM tip and 
extended until the sample detached from the cantilever. The resulting force-extension curves of the full-length native perlecan showed a single detachment peak with no noticeable unfolding events (see Fig. 4 ) regardless of where the tip attached to the proteoglycan. For SMFMs, force curves follow a similar trace pattern, where the last peak represents the rupture of the tipsample interaction. This detachment peak is distinguished from peaks that represent unfolding processes, in that after the sample-tip interaction is lost the tip snaps back to its starting neutral position and thus the force returns to baseline levels. We fit the force-extension curves to the eWLC model, which predicts perlecan is extended beyond its resting contour length, given the relative high forces ( 150 pN, Fig. 4).
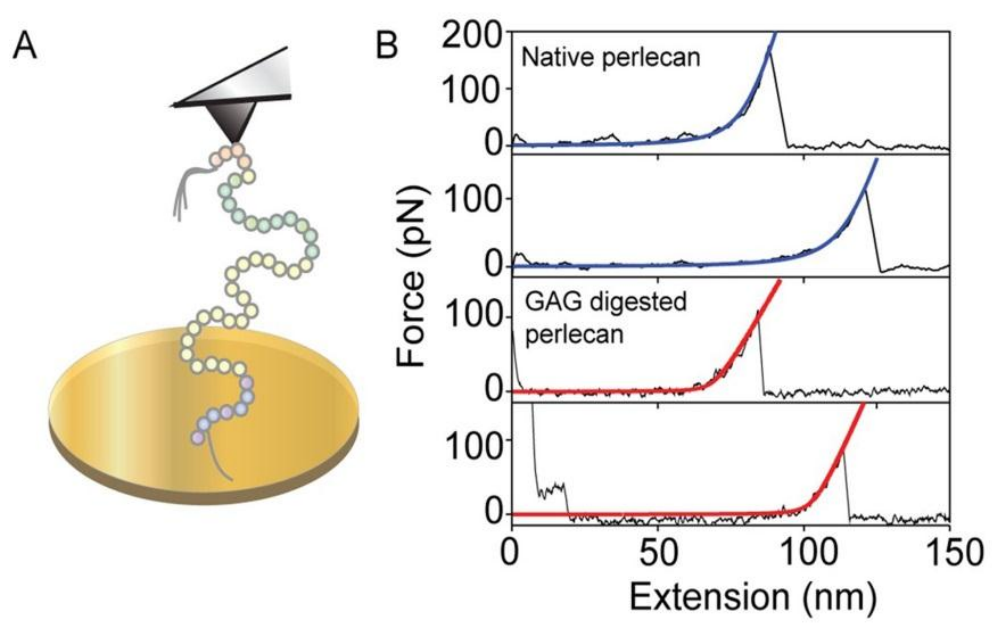

Figure 4. Stretching native full-length perlecan. (A) Purified perlecan was adsorbed onto a gold substrate and brought into contact with an AFM tip. As the stage moved away from the tip the perlecan molecule was pulled while force was recorded. (B) Two representative forceextension curves of pulling events of native perlecan and the GAG digested form with eWLC fitting in solid lines. The measured extension values in the force-extension curves ranged from 40-445 $\mathrm{nm}$ for native perlecan and 23-279 $\mathrm{nm}$ for the perlecan core. For both native perlecan and the GAG digested form there was only one peak observed. No unfolding occurred in either case.

The most probable values, resulting from Gaussian fits to histogram distributions, are $L_{p}$ $=1.2 \mathrm{~nm}$, and $K=2000 \mathrm{pN}$ (Fig. $5 A$ and $B$ ). To examine the core protein exclusively and ensure the measurements were not associated with the GAG portion of the proteoglycan, we 
completely removed the GAGs by digesting perlecan with heparitinase I, II, III and chondroitinase $\mathrm{ABC}$ and repeated the pulling analysis. We found that the profile of the force curves resembled what we saw in full-length perlecan, displaying a single detachment. When we fit the data to the eWLC model, we observed the core protein of perlecan to have $L_{p}=22$ $\mathrm{nm}$ and $K=890 \mathrm{pN}$ (Fig. $5 C$ and $D$ ). The $L_{p}=22 \mathrm{~nm}$ of the perlecan core from pulling is consistent with the $L_{p}=19 \mathrm{~nm}$ obtained from imaging. However, full-length perlecan had a $L_{p}$ $=1.2$, much lower than that of the core.
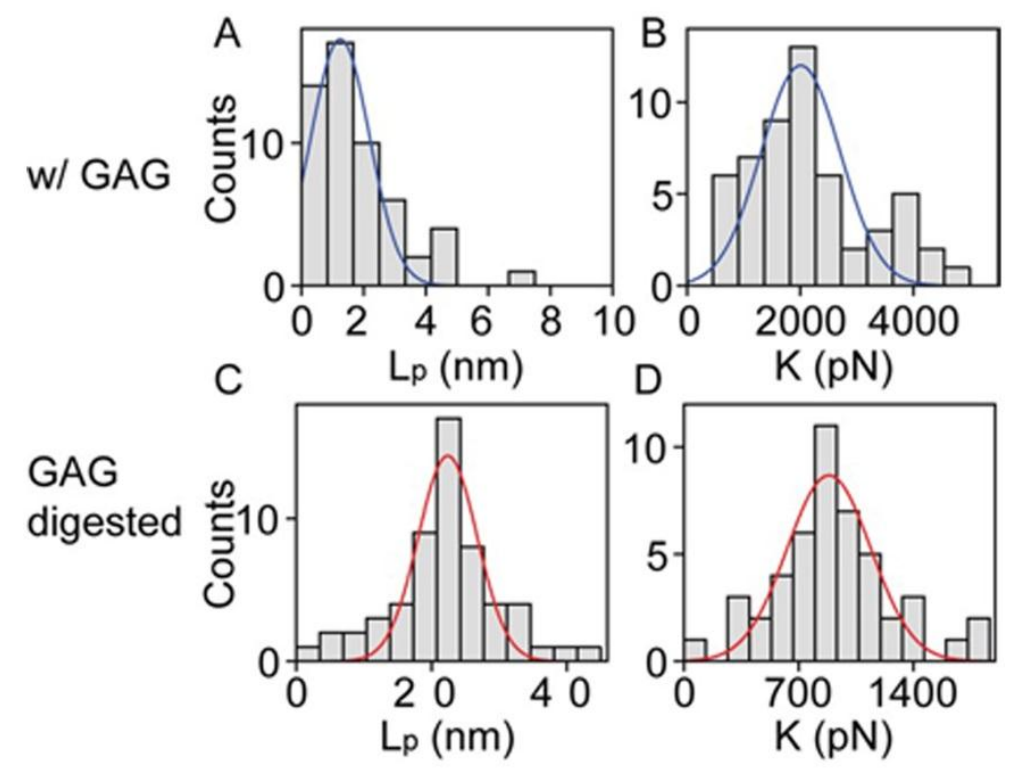

Figure 5. Elasticity of full-length perlecan with and without its GAG side chains. Histograms showing (A) native perlecan having a $L_{p}$ of $1.2 \mathrm{~nm}$ and (B) a $K$ of $2000 \mathrm{pN}$ from fitting to a Gaussian distribution. Digesting away the GAG chains increased perlecan's $L_{p}$ to 22 $\mathrm{nm}(\mathrm{C})$ and decreased $K$ value $(890 \mathrm{pN})(\mathrm{D})$.

In the experiments above, no module unfolding with the characteristic sequence of multiple peaks was observed, perhaps due to the presence of intradomain disulfide bonds. To confirm this, we added DTT to break the disulfide bonds of perlecan, to enable the forceinduced unfolding. When $1 \mathrm{mM}$ DTT was added, the force-extension curves exhibited 
sequences of sawtooth pattern, characteristic of the sequential unfolding of the protein modules [25,35,36] (see Fig. 6 A). The force curves were fitted with an inextensible WLC model (Eq. 3). Fig. $6 \mathrm{~B}$ shows that from the Gaussian fits to the histogram, the unfolding force with the highest occurrence frequency was $110 \mathrm{pN}$. The histogram of the change in contour length $\Delta L_{c}$, has a peak at $35 \mathrm{~nm}$ (Fig. 6 C). The observed unfolding forces of disulfide-cleaved perlecan are within the range of forces required to unfold elastic behaving proteins $[25,35,36]$. Under normal circumstances, perlecan exists as a stable intact protein, and only when chemically denatured by oxidizing the disulfides did perlecan unfold under tensile mechanical stress.
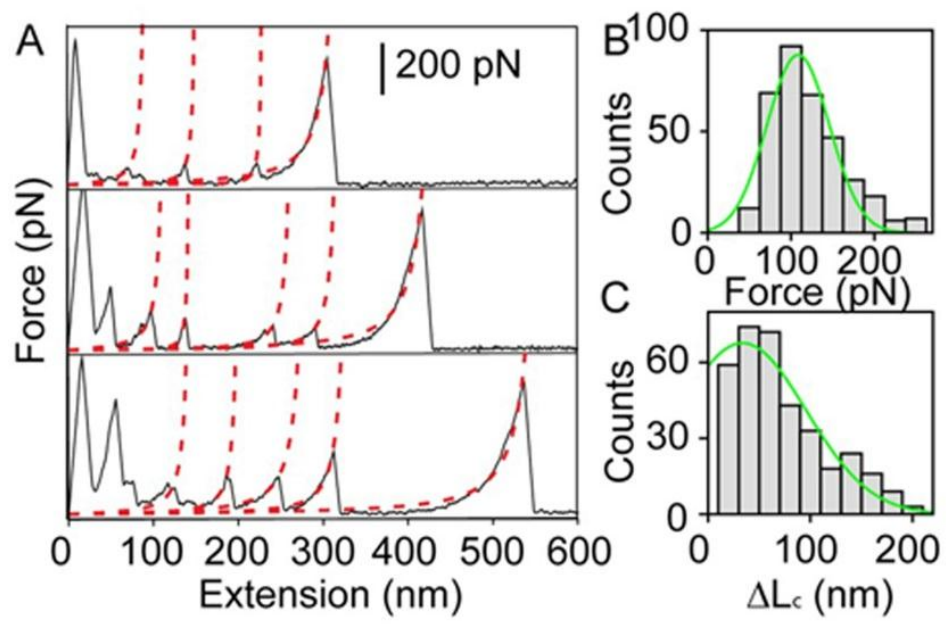

Figure 6. Perlecan was unfold only after being pretreated with a denaturing agent. (A) In the presence of DTT, multiple unfolding peaks were observed. Large initial peaks are due to non-specific attraction forces that cause tip to snap-in to the surface and therefore are excluded from data analysis. These peaks were fitted to the inextensible WLC model (dashed lines) to obtain the unfolding force. Note that the last peaks were usually as high as $500 \mathrm{pN}$. (B) The histogram of the unfolding force, fitted with a Gaussian distribution (solid line), shows a peak at $110 \mathrm{pN}$. (C) The histogram of the change in contour length between the unfolded peaks, $\Delta L_{c}$, shows a peak at $35 \mathrm{~nm}$.

\section{Discussion}


Our AFM data revealed that perlecan has a long and strong core protein that resists conformational changes when under mechanical distress. This result supports our previous hypothesis that perlecan possesses the physical properties to serve as a transverse tether in the LCS to sense fluid drag caused by mechanical loading [16]. We measured the unstressed end-to-end length of the perlecan core protein $(\sim 170 \mathrm{~nm})$ to demonstrate that perlecan was of sufficient size to transverse the annular gap ( 100 nm) and tether the osteocyte cell body to the canalicular wall [37]. In addition, we demonstrated the mechanical strength of perlecan core protein through single molecule pulling. Compared to the stretch modulus of the elastic protein titin [38], the stretch modulus $\mathrm{K}$ of perlecan core protein was found to be almost 5 times greater $(890 \mathrm{pN})$. Assuming the perlecan core protein as a rod with a cross-sectional area A (diameter of $4 \mathrm{~nm}$, Fig. 2), the tensile modulus of the core protein $E=K / A=71 \mathrm{MPa}$, which are smaller than those of collagen I fibril (<5 GPa, [39]) and actin filaments (1.8 GPa, [40]) but much larger than that of focal adhesion (5.5 kPa, [41]).

For perlecan to function as a viable osteocyte cell tether, it must be able to traverse the pericellular space and endure fluid flow drag forces. AFM images were taken to obtain an accurate measure of the true length native purified monomeric perlecan. Previously measurements of various aspects of perlecan's dimensions and molecular topography have been reported, including one study finding the core to be around $80 \mathrm{~nm}$ in length and each GAG attachment to extend up to $170 \mathrm{~nm}$ [42]. In another study, AFM imaging was used to characterize the size of perlecan, revealing two molecular volumes of the protein core at 290 $\mathrm{nm}^{3}$ and $710 \mathrm{~nm}^{3}$ [43]. Our results differ from previous studies, possibly due to the difference in the source perlecan and isolation method. We found on average, measured by AFM images, that perlecan extended about $170 \mathrm{~nm}$ in length (Fig. 2), agreeing with our past prediction based upon composite of detailed rotary shadowing electron microscopy and AFM images of recombinant domains [4]. Variations can be attributed to perlecan's flexible nature and ability to fold onto itself in variety of ways. In addition, it must be considered that adsorbed proteins can 
undertake substrate-induced conformations. Despite these uncertainties, our measurements of "resting" linear dimensions lead us to conclude that a single perlecan monomer easily can span the entire pericellular space, which has been measured to be about $100 \mathrm{~nm}$ wide, from osteocyte cell body to the mineralized bone surface [44].

To determine if perlecan is strong enough to handle mechanical loading, we estimated the fluid drag forces under normal loading conditions (400 microstrain in mouse tibia) to be 18.4 $\mathrm{pN}$ over one micron length of cell process [16]. This translates to less than $1 \mathrm{pN}$ of drag force acting transversely on each tether and less than $10 \mathrm{pN}$ tension along the long axis of the core protein, assuming the tether anchors at the bone wall and cell membrane at an angle larger than 2.8 degree to the radial direction of the canaliculus. This anchoring angle can be easily achieved if the effective length of the tether is no shorter than $100.04 \mathrm{~nm}$ to span the $100 \mathrm{~nm}$ gap between the cell membrane and the wall (Fig. 7). Our data suggest that perlecan is strong enough to sustain the tensional forces under physiological loading conditions ( $10 \mathrm{pN}$ for 400 microstrain loading), given its much higher rupture forces ( 150 pN, Fig. 4) under AFM pulling. The high end of physiology strain is about 2000 microstrain, resulting 5 times of the fluid drag, which is still lower than the rupture force measured for the perlecan core protein. In the pulling experiments, it was impossible to control the exact locations that the perlecan monomer anchored to the AFM tip and the substrate. Thus, the final rupture might occur at either end of the contact regions or along perlecan's linear structure including protein core and GAG side chain. However, unfolding or rupturing the protein core was highly unlikely under the testing condition. We did not observe the sequential peaks that are characteristic of protein unfolding and we only observed single peaks as the full-length perlecan was pulled (Fig. 4). Protein core unfolding could be observed after treatment with DTT to break the disulfide bonds (Fig. 6). Even under this denatured condition, the mean force to unfold perlecan was at the level of $110 \mathrm{pN}$ and the final rupture forces could be as high as more than $500 \mathrm{pN}$ (see the last peaks in Fig. 6 
A). These data led us to conclude that the perlecan protein core can easily sustain tensions at the order of $10 \mathrm{pN}$ under physiological loading conditions of the bone LCS.

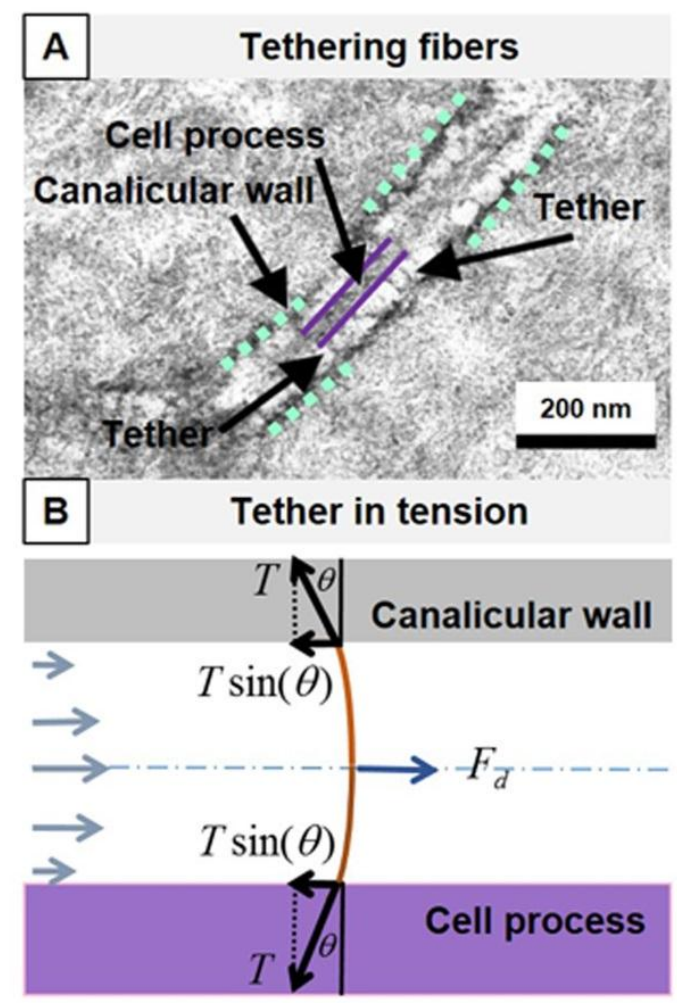

Figure 7. Perlecan tether under tension caused by fluid flow in the bone LCS. (A) A TEM image showing the transverse tethers span the space $(\sim 100 \mathrm{~nm})$ between cell process membrane and canalicular wall. (B) The fluid drag force $\left(F_{d}=\sim 1 \mathrm{pN}\right)$ along the axial direction induces a tension $(T=\sim 10 \mathrm{pN})$ within the tethering fiber, assuming the tether anchors at a small angle $\left(\theta=2.87^{\circ}\right)$ as suggested in $(A)$. The projection of the tension forces balances with the drag force $\left(2 T \sin (\theta)=F_{d}\right)$. The tension in the tether depends on the fluid drag force and the anchoring angle. Larger angles are anticipated to be associated with smaller tension forces under a given fluid drag force.

As a potential mechanosensing tether, we must compare the strength of perlecan itself to those of the anchors that it forms with the osteocyte cell membrane and bone matrix. One possibility is that perlecan interacts with the mineralized bone via its GAG side chains of domain I. The bone matrix is rich in collagen I, which is involved in heparin binding. It has been shown that both soluble and cell surface heparan sulfate proteoglycans bind with high affinity to native interstitial collagen I fibrils, with Kds approximately $10^{-10} \mathrm{M}$ and $10^{-9} \mathrm{M}$, respectively $[45,46]$. Our 
lab has previously shown that perlecan domain I can bind directly to collagen I monomers and fibrils, via its heparan sulfate side chains [47]. The cleavage of perlecan's domain I GAG chains with heparinases, but not chondroitinase, completely prevented binding to collagen I (PMID 15738663). Single molecule approaches have been used to measure the binding strength of heparan sulfate, with one study finding that as high as $310 \mathrm{pN}$ was required to rupture a multivalent heparan sulfate-ligand interaction [48]. This high strength of GAG-ligand interaction may explain our observations that perlecan with GAG showed a shorter persistent length $L_{p}$ $(1.2 \mathrm{~nm}$ vs. $22 \mathrm{~nm})$ and a higher stretch elasticity $K(2000 \mathrm{vs} .890 \mathrm{pN})$ than perlecan devoid of GAG (Fig. 5). It has been shown that perlecan links together the laminin and collagen IV rich networks of the epidermal BM in a spot-weld-like manner [49]. Perlecan's heparan sulfate chains are integrated into collagen network, which is likely how perlecan interacts with the mineralized bone wall of the LCS. Separating these two perlecan-linked independent networks requires repeated application of mechanical force. Unfortunately, the total amount of force required to break the perlecan linkage was not determined. In any case, the forces required to separate the bound protein-protein and protein-carbohydrate pairs (190-400 pN) [50-52] tend to be well above the forces exerted by fluid drag in the LCS $(10 \mathrm{pN})$. If indeed domain I is the attachment point to bone surface, it is most likely the opposing C-terminal end of perlecan (domains IV-V) is anchored to the osteocyte cell surface. Domain V interacts with cells surface integrins, most notably $\beta 1$ integrin [53]. Interestingly, $\beta 1$ integrin was shown to be involved in bone mechanotransduction, whereupon the knockout of $\beta 1$ integrin in cortical osteocytes limited bone loss resulting from disuse [54]. Domain IV also serves as a likely link for cell attachment, considering its length (nearly half of the perlecan) and its multiple repeats of Ig motifs (Fig. 1), which are often recognized in cell adhesion processes [55]. It is also possible that the perlecan tether may be indirectly linked to the osteocyte surface through a PCM-cell membrane complex. Despite the lack of mechanical measurements of the anchors at the two ends of the PCM tether, 
perlecan's ability to bind and link together various ECM proteins supports further investigations of the possible tether configurations in the bone LCS.

There are several limitations for our study. In many AFM single molecule studies, small recombinant proteins are often used with a priori known unfolding peak patterns. With perlecan, the force-extension profile was unknown, raising the concern whether or not our AFM pulling scheme was capable of detecting or causing any unfolding events. We thus destabilized the disulfide bonds of perlecan, making it more vulnerable to force-induce unfolding (Fig. 6). This set of experiments increased our confidence that the lack of multiple peaks during pulling perlecan and perlecan core protein indicates that there was no protein unfolding under the testing condition. Another limitation was that we were unable to control the exact probe attachment points during the pulling experiments. Ideally, we would have the most terminal ends of the protein attached to substrate and AFM tip. This would allow us to capture the stretch profile of the entire molecule. Functionalized substrate and AFM tip may help to achieve this idealized testing configuration.

In this study, AFM was used to capture images of perlecan monomers at nanoscale resolution and to perform single molecule force measurements. We found that the core protein of purified full-length human perlecan is of suitable size to span the pericellular space of the LCS, with a measured end-to-end length of $170 \pm 20 \mathrm{~nm}$ and a diameter of $2-4 \mathrm{~nm}$. Force pulling revealed a strong protein core that can withstand over $100 \mathrm{pN}$ of tension, well over the drag forces that are estimated to be exerted on the individual osteocyte tethers. Data fitting with an extensible worm-like chain model showed that the perlecan protein core has a mean elastic constant of $890 \mathrm{pN}$ and a corresponding Young's modulus of $71 \mathrm{MPa}$. We conclude perlecan has the physical properties that would allow it to act as a strong but elastic tether in the LCS.

\section{Experimental Procedures}

\subsection{Perlecan purification from HT-29 conditioned media}


HT-29, a human colon carcinoma cell line, formerly called WiDR, was chosen because these cells produce perlecan as their primary HSPG $[56,57]$. Cells were purchased from the American Type Culture Collection (Cat. No. CCL-218, ATCC, Manassas, VA) and cultured as described previously $[56,58]$. When cells were nearly $100 \%$ confluent as judged by eye in Corning Hyperflasks, they were switched to $2 \%(\mathrm{v} / \mathrm{v})$ fetal bovine serum (FBS) medium, and medium was collected and processed with protease inhibitors as recently described [58]. The solutions were processed through a S34100 Amicon spiral wound $100 \mathrm{kDa}$ molecular weight cutoff media concentrator cartridge. The resulting high molecular weight concentrated solution was subjected to diethyl aminoethyl (DEAE) anion exchange chromatography (GE Healthcare, Pittsburgh, PA). Equilibration buffer contained $2 \mathrm{M}$ urea, $50 \mathrm{mM}$ PIPES (pH 7.0), $250 \mathrm{mM} \mathrm{NaCl}$, $2.5 \mathrm{mM}$ EDTA, $0.5 \mathrm{mM}$ benzamadine, $0.5 \mathrm{mM}$ phenylmethyl sulfonylfluoride (PMSF), and $0.02 \%$ (w/v) sodium azide. Elution buffer was identical to equilibration buffer except the $\mathrm{NaCl}$ was 750 $\mathrm{mM}$. Eluted $1.5 \mathrm{~mL}$ fractions were collected and the absorbance at $280 \mathrm{~nm}$ read. Fractions were individually analyzed by dot blot immunoassay with anti-perlecan Dm IV antibody A7LC (described below). Pooled fractions containing perlecan were dialyzed in MilliQ water and centrifuged in a speed vac until the desired concentration was obtained. The resulting perlecan enriched solution was separated by Sepharose CL-4B (Sigma-Aldrich, St. Louis, MO) gel filtration chromatography in the presence of $0.8 \mathrm{M} \mathrm{NaCl}$ phosphate buffered saline (PBS, pH 7.4) buffer. Perlecan peak containing fractions were pooled dialyzed in MilliQ water and centrifuged in a speed vac. Perlecan pools were subjected to gradient heparin Sepharose 6 fast flow (GE Healthcare) chromatography in PBS containing $0.2,0.3,0.5,1.0$, and $1.5 \mathrm{M} \mathrm{NaCl}$. Perlecan, as determined by A7LC dot blot, eluted at $0.3 \mathrm{M} \mathrm{NaCl}$. Perlecan was centrifuged in a speed vac and dialyzed in a final PBS buffer. Samples were aliquotted and stored at $-80^{\circ} \mathrm{C}$. Purity was assessed by silver stain as described below.

\subsection{Cleavage of glycosaminoglycan (GAG) side chains}


To investigate the elastic properties of the core protein exclusively, perlecan's GAG side chains were cleaved by incubating the molecule with heparitinase I, II, III and chondroitinase $\mathrm{ABC}$ in PBS with $2 \mathrm{mM}$ calcium chloride at 0.1 units per enzyme at $37^{\circ} \mathrm{C}$ overnight. Enzymes were separated from the core protein by passing samples through an anti-perlecan A74 antibody (a generous gift from John M. Whitelock) affinity column following established protocols (26). All final perlecan containing fractions were pooled, dialyzed in MilliQ water and centrifuged in a speed vac. The A7L6C (Sigma-Aldrich) antibody was used for all dot-blot and western blot analyses. Purity and removal of GAG chains was confirmed by collapse seen via silver stain. Heparan sulfate from bovine kidney was purchased from Sigma-Aldrich and used as a control for AFM pulling.

\subsection{Dot blot and silver stain conditions}

Purified full-length and GAG digested perlecan were denatured at $99^{\circ} \mathrm{C}$ with reducing sample buffer (final $60 \mathrm{mM}$ Tris- $\mathrm{HCl}, 1 \%$ (w/v) SDS, 10\% glycerol, 2\% (v/v) 2-mercaptoethanol, pH 6.8 with tracking dye) for 5-10 min. Samples were separated with SDS-polyacrylamide gel electrophoresis (PAGE) in $1 \mathrm{~mm}$ Novex NuPAGE 4-12\% acrylamide gradient Bis-Tris buffered gels (Invitrogen, Carlsbad, CA) in a Novex NuPAGE system (Invitrogen) with $1 \times$ MOPS SDS buffer (Invitrogen) at 150 constant volts for approximately $80 \mathrm{~min}$. SeeBlue® Plus2 molecular weight marker (Invitrogen) was used to determine molecular weight. Silverstains were performed with the Silverquest Silver Stain kit according to manufacturer's directions (Invitrogen). For perlecan dot blots, $20 \mu \mathrm{L}$ of sample was added to a Bio-Rad slot blot apparatus (Cat. No. 170-6545) and allowed to bind pre-PBS rinsed $0.45 \mu \mathrm{m}$ nitrocellulose membrane for $2 \mathrm{~h}$ at room temperature. After vacuum suction, the dot blot was blocked in a 3\% (w/v) BSA Tris buffered saline with $0.05 \%(\mathrm{v} / \mathrm{v})$ Tween-20 (TBST) for $2 \mathrm{~h}$ at room temperature. A7LC antibody (Invitrogen) at 1:5,000 was incubated in the blocking solution overnight on a $4^{\circ} \mathrm{C}$ shaker. After 3 by 5 min TBST washes, the membranes were incubated with 1:200,000 goat anti-rat HRP 
conjugated antibody in 3\% BSA TBST for $2 \mathrm{~h}$ at room temperature. Blots were washed again and incubated with chemiluminescence substrate (West Dura Extended Substrate, Pierce, Rockford, IL) for 5 min before being exposed to film.

\subsection{AFM experiments}

For all AFM studies, data was gathered on a Nanoscope $V$ with Multimode 8 AFM (Bruker AXS, Santa Barbara, CA). The spring constant of each cantilever was calibrated prior to each experimental run according to the vendor's instructions. The cantilever deflectionsensitivity was calculated from a force curve generated by pressing the tip against the gold substrate in either air or PBS solution. Once sensitivity was determined, the thermal tune method was used to calculate the cantilever spring constant. For this method, the tip retracted from the substrate surface and oscillated to identify the correct resonance frequency.

For imaging experiments, discs of mica were glued to steel discs with epoxy and allowed to dry overnight. The dried mica disks were cleaved with Scotch tape to expose a fresh surface. 10 $\mu \mathrm{L}$ of perlecan samples $(10 \mu \mathrm{g} / \mathrm{mL}$ ) were left on mica for 5 min, washed with double distilled water and then dried with nitrogen air. Imaging was carried out in ScanAsyst mode at room temperature using ScanAsyst-Air tips (spring constant: $0.4 \mathrm{~N} / \mathrm{m}$ and radius: $2 \mathrm{~nm}$, Bruker AXS). A total of 27 AFM images of full-length perlecan were traced using ImageJ (National Institutes of Health, Bethesda, MD) [59] and Engauge digitizer software (Free Software Foundation). A program written in MATLAB (The MathWorks, Natick, MA) was used to interpolate the $x y$

coordinates of the perlecan contours and calculate the persistence length $\left(L_{p}\right)$ of perlecan using a tangent to tangent correlation function [60],

$$
\langle\cos (\theta)\rangle=\exp \left(-L / 2 L_{p}\right),(\text { Eq. } 1)
$$

The $L_{p}$ is a measure of bending stiffness of a polymer or linear molecule, with a high $L_{p}$ conferring low flexibility. $L_{p}$ is defined as the distance over which the correlation of tangents is 
lost, among two points along the contour trace. The angle, $\theta$ is that between two tangent vectors at a pair of points separated by a given length $(L)$.

Pulling experiments were performed in solution under contact mode using a calibrated MLCT-D tips (spring constant: $0.01 \mathrm{~N} / \mathrm{m}$ and radius: $20 \mathrm{~nm}$, Bruker AXS). MLCT probes have an intentionally dull tip ideal for force measurements and imaging extremely delicate samples in fluid. Perlecan at $20 \mathrm{nM}$ in PBS was incubated at $37^{\circ} \mathrm{C}$ prior to being deposited onto a fresh gold substrate at room temperature for 10-15 min. The AFM tip was brought in contact with the surface for 1-3 s to allow the perlecan molecule to attach to the silicon nitride cantilever tip. All of the force measurements were obtained in aqueous PBS (pH 7.4) with a pulling velocity of $1000 \mathrm{~nm} / \mathrm{s}$. Aggregation of perlecan molecules was minimized by using a low protein sample concentration as previously described [43]. At this diluted concentration (one perlecan molecule per $0.8 \mu \mathrm{m}^{3}$ ), the fluid volume displaced by the tip (radius of $20 \mathrm{~nm}$ ) moving $10-100 \mu \mathrm{m}$ into the fluid was anticipated to be approximately $0.01-0.1 \mu \mathrm{m}^{3}$. Thus, it was unlikely to have multiple perlecan molecules attached to the tip and the recorded forces were most likely due to single molecule engagements.

For module unfolding experiments, $1 \mu \mathrm{L}$ of $10 \mathrm{mM}$ of dithiothreitol (DTT) was added to $10 \mu \mathrm{L}$ of perlecan solution and incubated as described above, and force measurements were taken in $1 \mathrm{mM}$ of PBS and DTT buffer solution. Each experiment lasted from 1-3 $\mathrm{h}$.

To obtain successful tip-sample engagement, for each experimental condition roughly 500-3000 force measurements were performed. In total, 54 native full-length, 57 GAG digested, and 345 DTT treated perlecan force curves were collected for data analysis. Pulling events capturing multiple tip interactions or oligomers were excluded from data analysis by selecting curves with only one detachment peak and of extension length similar to a single perlecan monomer. The force curves were fitted with either the extensible WLC (eWLC) model $[61,62]$ (for native perlecan with or without GAG), 


$$
x=L_{c}\left[1-\frac{1}{\left(4 \beta L_{p} F\right)^{1 / 2}}+\frac{F}{K}\right]
$$

or inextensible WLC model [61,63-65] (for denatured DTT treated perlecan),

$$
F(x)=\frac{1}{L_{p} \beta}\left[\frac{1}{4\left(1-x / L_{c}\right)^{2}}+\frac{x}{L_{c}}-\frac{1}{4}\right],
$$

where $x$ is the extension, $F$ is the force, $K$ is the elastic stretch modulus and $\beta=1 / k_{B} T$

where $k_{B}$ is the Boltzmann constant, $T$ is the temperature, $L_{c}$ is the contour length of the total end-to-end length of an extended molecule and $L_{p}$ is the persistence length. Histograms of the $L_{c}$ and $L_{p}$ distributions were fitted to a Gaussian curve to get statistical measures of the two parameters. For unfolding studies, peak-to-peak distance was measured as change in contour length ( $\Delta L_{c}$ ), which reflects the size of a particular region of the protein core that is unfolding. Equation 2 (eWLC) was used to model conditions where the protein remain folded, whereas, the inextensible WLC model (Eq. 3) is more suitable for protein unfolding and therefore used for denatured conditions.

\section{Acknowledgments}

This work was supported by grants from National Science Foundation (0907676), Welch Foundation (C-1632), the Keck Center Nanobiology Interdisciplinary Graduate Training Program of the Gulf Coast Consortia, National Institutes of Health (R01AR054385 and P01CA098912, T32EB009379), and Rice University Alliances for Graduate Education and the Professoriate. The authors would like to thank Dr. William R. Thompson for many helpful discussions, and Drs. Daniel A. Harrington and Danielle Wu for their help editing the manuscript.

\section{References}

1. Iozzo, R. V. \& Schaefer, L. (2015). Proteoglycan form and function: A comprehensive nomenclature of proteoglycans. Matrix Biol. 42, 11-55.

2. Farach-Carson, M. C., Warren, C. R., Harrington, D. A. \& Carson, D. D. (2014). Border patrol: insights into the unique role of perlecan/heparan sulfate proteoglycan 2 at cell and tissue borders. Matrix Biol. 34, 64-79.

3. Iozzo, R. V., Cohen, I. R., Grassel, S. \& Murdoch, A. D. (1994). The biology of perlecan: the multifaceted heparan sulphate proteoglycan of basement 
membranes and pericellular matrices. Biochem. J. 302 ( Pt 3), 625-639.

4. Farach-Carson, M. C. \& Carson, D. D. (2007). Perlecan - a multifunctional extracellular proteoglycan scaffold. Glycobiology 17, 897-905.

5. Warren, C. R., Kassir, E., Spurlin, J., Martinez, J., Putnam, N. H. \& FarachCarson, M. C. (2015). Evolution of the perlecan/HSPG2 gene and its activation in regenerating Nematostella vectensis. PLoS One 10, e0124578.

6. Kanwar, Y. S., Veis, A., Kimura, J. H. \& Jakubowski, M. L. (1984). Characterization of heparan sulfate-proteoglycan of glomerular basement membranes. Proc. Natl. Acad. Sci. U. S. A. 81, 762-766.

7. Rogalski, T. M., Mullen, G. P., Bush, J. A., Gilchrist, E. J. \& Moerman, D. G. (2001). UNC-52/perlecan isoform diversity and function in Caenorhabditis elegans. Biochem. Soc. Trans. 29, 171-176.

8. Brown, A. J., Alicknavitch, M., D'Souza, S. S., Daikoku, T., Kirn-Safran, C. B., Marchetti, D., Carson, D. D. \& Farach-Carson, M. C. (2008). Heparanase expression and activity influences chondrogenic and osteogenic processes during endochondral bone formation. Bone 43, 689-699.

9. Costell, M., Gustafsson, E., Aszodi, A., Morgelin, M., Bloch, W., Hunziker, E., Addicks, K., Timpl, R. \& Fassler, R. (1999). Perlecan maintains the integrity of cartilage and some basement membranes. J. Cell Biol. 147, 1109-1122.

10. Xu, Z., Ichikawa, N., Kosaki, K., Yamada, Y., Sasaki, T., Sakai, L. Y., Kurosawa, H., Hattori, N. \& Arikawa-Hirasawa, E. (2010). Perlecan deficiency causes muscle hypertrophy, a decrease in myostatin expression, and changes in muscle fiber composition. Matrix Biol. 29, 461-470.

11. Ning, L., Xu, Z., Furuya, N., Nonaka, R., Yamada, Y. \& Arikawa-Hirasawa, E. (2015). Perlecan inhibits autophagy to maintain muscle homeostasis in mouse soleus muscle. Matrix Biol.

12. Wilusz, R. E., Sanchez-Adams, J. \& Guilak, F. (2014). The structure and function of the pericellular matrix of articular cartilage. Matrix Biol. 39, 25-32.

13. Vincent, T. L., McLean, C. J., Full, L. E., Peston, D. \& Saklatvala, J. (2007). FGF2 is bound to perlecan in the pericellular matrix of articular cartilage, where it acts as a chondrocyte mechanotransducer. Osteoarthr. Cartil. 15, 752-763.

14. Prein, C., Warmbold, N., Farkas, Z., Schieker, M., Aszodi, A. \& ClausenSchaumann, H. (2015). Structural and mechanical properties of the proliferative zone of the developing murine growth plate cartilage assessed by atomic force microscopy. Matrix Biol.

15. Thompson, W. R., Modla, S., Grindel, B. J., Czymmek, K. J., Kirn-Safran, C. B., Wang, L., Duncan, R. L. \& Farach-Carson, M. C. (2011). Perlecan/Hspg2 deficiency alters the pericellular space of the lacunocanalicular system surrounding osteocytic processes in cortical bone. J. Bone Miner. Res. 26, 618629.

16. Wang, B., Lai, X., Price, C., Thompson, W. R., Li, W., Quabili, T. R., Tseng, W. J., Liu, X. S., Zhang, H., Pan, J., Kirn-Safran, C. B., Farach-Carson, M. C. \& Wang, L. (2014). Perlecan-containing pericellular matrix regulates solute transport and mechanosensing within the osteocyte lacunar-canalicular system. J. Bone Miner. Res. 29, 878-891.

17. Fritton, S. P. \& Weinbaum, S. (2009). Fluid and Solute Transport in Bone: Flow- 
Induced Mechanotransduction. Annu. Rev. Fluid Mech. 41, 347-374.

18. Weinbaum, S., Cowin, S. C. \& Zeng, Y. (1994). A model for the excitation of osteocytes by mechanical loading-induced bone fluid shear stresses. J. Biomech. 27, 339-360.

19. Burger, E. H., Klein-Nulend, J., van der Plas, A. \& Nijweide, P. J. (1995). Function of osteocytes in bone--their role in mechanotransduction. J. Nutr. 125, 2020S-2023S.

20. Dallas, S. L., Prideaux, M. \& Bonewald, L. F. (2013). The osteocyte: an endocrine cell ... and more. Endocr. Rev. 34, 658-690.

21. Klein-Nulend, J., Bakker, A. D., Bacabac, R. G., Vatsa, A. \& Weinbaum, S. (2013). Mechanosensation and transduction in osteocytes. Bone 54, 182-190.

22. Bonewald, L. F. (2011). The amazing osteocyte. J. Bone Miner. Res. 26, 229-

238.

23. You, L., Cowin, S. C., Schaffler, M. B. \& Weinbaum, S. (2001). A model for strain amplification in the actin cytoskeleton of osteocytes due to fluid drag on pericellular matrix. J. Biomech. 34, 1375-1386.

24. Neuman, K. C. \& Nagy, A. (2008). Single-molecule force spectroscopy: optical tweezers, magnetic tweezers and atomic force microscopy. Nat. Methods 5, 491505.

25. Rief, M., Gautel, M., Oesterhelt, F., Fernandez, J. M. \& Gaub, H. E. (1997). Reversible unfolding of individual titin immunoglobulin domains by AFM. Science 276, 1109-1112.

26. Marszalek, P. E., Lu, H., Li, H. B., Carrion-Vazquez, M., Oberhauser, A. F., Schulten, K. \& Fernandez, J. M. (1999). Mechanical unfolding intermediates in titin modules. Nature 402, 100-103.

27. Harris, N. C., Song, Y. \& Kiang, C. H. (2007). Experimental free energy surface reconstruction from single-molecule force spectroscopy using Jarzynski's equality. Phys. Rev. Lett. 99, 068101.

28. Wijeratne, S. S., Botello, E., Yeh, H. C., Zhou, Z., Bergeron, A. L., Frey, E. W., Patel, J. M., Nolasco, L., Turner, N. A., Moake, J. L., Dong, J. F. \& Kiang, C. H. (2013). Mechanical activation of a multimeric adhesive protein through domain conformational change. Phys. Rev. Lett. 110, 108102.

29. Marin, J. L., Muniz, J., Huerta, M. \& Trujillo, X. (2003). Folding-unfolding of FN-III domains in tenascin: an elastically coupled two-state system. J. Biomech. 36, 1733-1737.

30. Liu, D., Ge, L., Wang, F., Takahashi, H., Wang, D., Guo, Z., Yoshimura, S. H., Ward, T., Ding, X., Takeyasu, K. \& Yao, X. (2007). Single-molecule detection of phosphorylation-induced plasticity changes during ezrin activation. FEBS Lett. 581, 3563-3571.

31. Kellermayer, M. S., Smith, S. B., Granzier, H. L. \& Bustamante, C. (1997). Folding-unfolding transitions in single titin molecules characterized with laser tweezers. Science 276, 1112-1116.

32. Li, H., Oberhauser, A. F., Fowler, S. B., Clarke, J. \& Fernandez, J. M. (2000). Atomic force microscopy reveals the mechanical design of a modular protein. Proc. Natl. Acad. Sci. U. S. A. 97, 6527-6531.

33. Wang, K., Forbes, J. G. \& Jin, A. J. (2001). Single molecule measurements of $t$ 
itin elasticity. Prog. Biophys. Mol. Biol. 77, 1-44.

34. Whitelock, J. M., Graham, L. D., Melrose, J., Murdoch, A. D., lozzo, R. V. \& Underwood, P. A. (1999). Human perlecan immunopurified from different endothelial cell sources has different adhesive properties for vascular cells. Matrix Biol. 18, 163-178.

35. Oberhauser, A. F., Marszalek, P. E., Erickson, H. P. \& Fernandez, J. M. (1998). The molecular elasticity of the extracellular matrix protein tenascin. Nature 393, 181-185.

36. Rief, M., Pascual, J., Saraste, M. \& Gaub, H. E. (1999). Single molecule force spectroscopy of spectrin repeats: Low unfolding forces in helix bundles. J. Mol. Biol. 286, 553-561.

37. You, L. D., Weinbaum, S., Cowin, S. C. \& Schaffler, M. B. (2004). Ultrastructure of the osteocyte process and its pericellular matrix. Anat. Rec. A Discov. Mol. Cell Evol. Biol. 278, 505-513.

38. Linke, W. A., Ivemeyer, M., Mundel, P., Stockmeier, M. R. \& Kolmerer, B. (1998). Nature of PEVK-titin elasticity in skeletal muscle. Proc. Natl. Acad. Sci. U. S. A. 95, 8052-8057.

39. Hofmann, H., Voss, T., Kuhn, K. \& Engel, J. (1984). Localization of flexible sites in thread-like molecules from electron micrographs. Comparison of interstitial, basement membrane and intima collagens. J. Mol. Biol. 172, 325-343.

40. Kojima, H., Ishijima, A. \& Yanagida, T. (1994). Direct measurement of stiffness of single actin filaments with and without tropomyosin by in vitro nanomanipulation. Proc. Natl. Acad. Sci. U. S. A. 91, 12962-12966.

41. Balaban, N. Q., Schwarz, U. S., Riveline, D., Goichberg, P., Tzur, G., Sabanay, I., Mahalu, D., Safran, S., Bershadsky, A., Addadi, L. \& Geiger, B. (2001). Force and focal adhesion assembly: a close relationship studied using elastic micropatterned substrates. Nat. Cell Biol. 3, 466-472.

42. Saku, T. \& Furthmayr, H. (1989). Characterization of the major heparan sulfate proteoglycan secreted by bovine aortic endothelial cells in culture. Homology to the large molecular weight molecule of basement membranes. J. Biol. Chem. 264, 3514-3523.

43. Chen, C. H. \& Hansma, H. G. (2000). Basement membrane macromolecules: insights from atomic force microscopy. J. Struct. Biol. 131, 44-55.

44. Lai, X., Price, C., Modla, S., Thompson, W. R., Caplan, J., Kirn-Safran, C. B. \& Wang, L. (2015). The dependences of osteocyte network on bone compartment, age, and disease. Bone Res. 3, 15009.

45. Koda, J. E. \& Bernfield, M. (1984). Heparan sulfate proteoglycans from mouse mammary epithelial cells. Basal extracellular proteoglycan binds specifically to native type I collagen fibrils. J. Biol. Chem. 259, 11763-11770.

46. Koda, J. E., Rapraeger, A. \& Bernfield, M. (1985). Heparan sulfate proteoglycans $f \quad$ rom mouse mammary epithelial cells. Cell surface proteoglycan as a receptor for interstitial collagens. J. Biol. Chem. 260, 8157-8162.

47. Yang, W. D., Gomes, R. R., Jr., Alicknavitch, M., Farach-Carson, M. C. \& Carson, D. D. (2005). Perlecan domain I promotes fibroblast growth factor 2 delivery in collagen I fibril scaffolds. Tissue Eng. 11, 76-89.

48. Guo, C., Fan, X., Qiu, H., Xiao, W., Wang, L. \& Xu, B. (2015). High-resolution 
probing heparan sulfate-antithrombin interaction on a single endothelial cell surface: single-molecule AFM studies. Phys. Chem. Chem. Phys. 17, 1330113306.

49. Behrens, D. T., Villone, D., Koch, M., Brunner, G., Sorokin, L., Robenek, H., Bruckner-Tuderman, L., Bruckner, P. \& Hansen, U. (2012). The epidermal basement membrane is a composite of separate laminin- or collagen IVcontaining networks connected by aggregated perlecan, but not by nidogens. J. Biol. Chem. 287, 18700-18709.

50. Dammer, U., Popescu, O., Wagner, P., Anselmetti, D., Guntherodt, H. J. \& Misevic, G. N. (1995). Binding strength between cell adhesion proteoglycans measured by atomic force microscopy. Science 267, 1173-1175.

51. Bell, G. I. (1978). Models for the specific adhesion of cells to cells. Science 200, 618-627.

52. Bucior, I., Scheuring, S., Engel, A. \& Burger, M. M. (2004). Carbohydratecarbohydrate interaction provides adhesion force and specificity for cellular recognition. J. Cell Biol. 165, 529-537.

53. Hayashi, K., Madri, J. A. \& Yurchenco, P. D. (1992). Endothelial cells interact with the core protein of basement membrane perlecan through beta 1 and beta 3 integrins: an adhesion modulated by glycosaminoglycan. J. Cell Biol. 119, 945959.

54. Phillips, J. A., Almeida, E. A., Hill, E. L., Aguirre, J. I., Rivera, M. F., Nachbandi, I., Wronski, T. J., van der Meulen, M. C. \& Globus, R. K. (2008). Role for beta1 integrins in cortical osteocytes during acute musculoskeletal disuse. Matrix Biol. 27, 609-618.

55. Wai Wong, C., Dye, D. E. \& Coombe, D. R. (2012). The role of immunoglobulin superfamily cell adhesion molecules in cancer metastasis. Int. J. Cell Biol. 2012, 340296.

56. Iozzo, R. V. (1984). Biosynthesis of heparan sulfate proteoglycan by human colon carcinoma cells and its localization at the cell surface. J. Cell Biol. 99, 403417.

57. Chen, T. R., Drabkowski, D., Hay, R. J., Macy, M. \& Peterson, W. (1987). WiDr is a derivative of another colon adenocarcinoma cell-line, HT-29. Cancer Genet. Cytogen. 27, 125-134.

58. Grindel, B. J., Martinez, J. R., Pennington, C. L., Muldoon, M., Stave, J., Chung, L. W. \& Farach-Carson, M. C. (2014). Matrilysin/matrix metalloproteinase7(MMP7) cleavage of perlecan/HSPG2 creates a molecular switch to alter prostate cancer cell behavior. Matrix Biol. 36, 64-76.

59. Schneider, C. A., Rasband, W. S. \& Eliceiri, K. W. (2012). NIH Image to ImageJ: 25 years of image analysis. Nat. Methods 9, 671-675.

60. Wiggins, P. A., van der Heijden, T., Moreno-Herrero, F., Spakowitz, A., Phillips, R., Widom, J., Dekker, C. \& Nelson, P. C. (2006). High flexibility of DNA on short length scales probed by atomic force microscopy. Nat. Nanotechnol. 1, 137-141.

61. Bustamante, C., Marko, J. F., Siggia, E. D. \& Smith, S. (1994). Entropic elasticity of lambda-phage DNA. Science 265, 1599-1600.

62. Calderon, C. P., Chen, W. H., Lin, K. J., Harris, N. C. \& Kiang, C. H. (2009). Quantifying DNA melting transitions using single-molecule force spectroscopy. J. 
Phys. Condens. Matter 21, 34114.

63. Bakajin, O. B., Duke, T. A. J., Chou, C. F., Chan, S. S., Austin, R. H. \& Cox, E. C. (1998). Electrohydrodynamic stretching of DNA in confined environments. Phys. Rev. Lett. 80, 2737-2740.

64. Onoa, B., Dumont, S., Liphardt, J., Smith, S. B., Tinoco, I. \& Bustamante, C. (2003). Identifying kinetic barriers to mechanical unfolding of the T-thermophila ribozyme. Science 299, 1892-1895.

65. Chen, W. S., Chen, W. H., Chen, Z., Gooding, A. A., Lin, K. J. \& Kiang, C. H. (2010). Direct observation of multiple pathways of single-stranded DNA stretching. Phys. Rev. Lett. 105, 218104. 